\title{
The Influence of Context on Distinct Facial Expressions of Disgust
}

Peter J. Reschke

Brigham Young University - Provo, peter_reschke@byu.edu

Eric A. Walle

University of California, Merced

Jennifer M. Knothe

University of California, Merced

Lukas D. Lopez

University of California, Merced

Follow this and additional works at: https://scholarsarchive.byu.edu/facpub

Part of the Other Social and Behavioral Sciences Commons

\section{Original Publication Citation}

Reschke, P. J., Walle, E., A, Knothe, J., M., \& Lopez, L. D. (2019). The influence of context on distinct facial expressions of disgust. Emotion, 19, 365-370.

\section{BYU ScholarsArchive Citation}

Reschke, Peter J.; Walle, Eric A.; Knothe, Jennifer M.; and Lopez, Lukas D., "The Influence of Context on Distinct Facial Expressions of Disgust" (2018). Faculty Publications. 4153.

https://scholarsarchive.byu.edu/facpub/4153 


\title{
BRIEF REPORT
}

\section{The Influence of Context on Distinct Facial Expressions of Disgust}

\author{
Peter J. Reschke, Eric A. Walle, Jennifer M. Knothe, and Lukas D. Lopez \\ University of California, Merced
}

\begin{abstract}
Face perception is susceptible to contextual influence and perceived physical similarities between emotion cues. However, studies often use structurally homogeneous facial expressions, making it difficult to explore how within-emotion variability in facial configuration affects emotion perception. This study examined the influence of context on the emotional perception of categorically identical, yet physically distinct, facial expressions of disgust. Participants categorized two perceptually distinct disgust facial expressions, "closed" (i.e., scrunched nose, closed mouth) and "open" (i.e., scrunched nose, open mouth, protruding tongue), that were embedded in contexts comprising emotion postures and scenes. Results demonstrated that the effect of nonfacial elements was significantly stronger for "open" disgust facial expressions than "closed" disgust facial expressions. These findings provide support that physical similarity within discrete categories of facial expressions is mutable and plays an important role in affective face perception.
\end{abstract}

Keywords: emotion perception, emotion, perceptual similarity, facial expression recognition, context

Supplemental materials: http://dx.doi.org/10.1037/emo0000445.supp

Facial expressions have long been emphasized as communicating discrete emotions (Ekman, 1993; Ekman \& O'Sullivan, 1988; Tomkins \& McCarter, 1964). However, research from the past decade demonstrates that context plays a significant role in emotion perception (e.g., Aviezer et al., 2008; Bliss-Moreau, Moadab, \& Machado, 2017; Meeren, van Heijnsbergen, \& de Gelder, 2005; Mondloch, Nelson, \& Horner, 2013; Reschke, Knothe, Lopez, \& Walle, 2018). The perceptual similarity hypothesis ${ }^{1}$ suggests that the perceived physical similarity between affective facial expressions is a fundamental determinant of the magnitude of contextual influence (see Hassin, Aviezer, \& Bentin, 2013). For example, a disgust facial expression embedded in an anger context (an emotion with a corresponding facial expression of high perceptual similarity with disgust) is more likely to be miscategorized as anger than if embedded in a fear context (an emotion with a corresponding facial expression of low perceptual similarity; Aviezer et al., 2008; Susskind, Littlewort, Bartlett, Movellan, \& Anderson, 2007). Research using prototypical facial expressions has provided evidence to support this phenomenon, (i.e., hereafter, confusability effect; Aviezer, Hassin, \& Bentin, 2012; Aviezer et al., 2008; Aviezer, Trope, \& Todorov, 2012; Reschke et al., 2018).

This article was published Online First June 11, 2018.

Peter J. Reschke, Eric A. Walle, Jennifer M. Knothe, and Lukas D. Lopez, Department of Psychological Sciences, University of California, Merced.

Correspondence concerning this article should be addressed to Peter J. Reschke, Department of Psychological Sciences, University of California, Merced, 5200 North Lake Road, Merced, CA 95343. E-mail: preschke@ ucmerced.edu
However, research has yet to examine whether within-face variability in physical configuration impacts categorization of facial expressions in context. More concretely, rather than comparing contextual influences on perceptually and categorically distinct facial expressions, a more stringent test of the perceptual similarity hypothesis would be to test facial expressions that are categorically equivalent (e.g., both convey disgust) but are physically distinct. The present investigation examined this more rigorous research question.

Facial expressions within the same discrete emotion category can include different combinations of action units (AUs). Consider disgust, a facial expression commonly used in studies examining the role of context on emotion perception (e.g., Aviezer et al., 2008; Reschke et al., 2018). A disgust facial expression can feature either AU 9 (wrinkled nose) and AU 10 (raised upper lip) or AU 6 (raised cheeks), AU 7 (tightened eyelids), AU 10 (raised upper lip), and AU 26 (dropped jaw; Widen, Pochedly, Pieloch, \& Russell, 2013; see also Calder et al., 2010; Rozin, Lowery, \& Ebert, 1994). Although categorically matching, these physically distinct displays of disgust may include expressive cues that are more confusable with other facial expressions, such as a dropped jaw in a fear expression (Ekman, 2003). Such structural flexibility within discrete facial expressions holds important implications for emotion perception. Specifically, the physical similarities between discrete facial expressions of emotion are variable and may result in differences in how contextual features influence emotion perception.

\footnotetext{
${ }^{1}$ Also referred to as the emotion seeds hypothesis (e.g., Aviezer et al., 2008; Mondloch et al., 2013).
} 
This study examined adult categorization of open- and closedmouth facial expressions of disgust embedded in emotion contexts (i.e., an emotion posture in an emotion scene). In line with prior research, we predicted that participant categorizations of disgust facial expressions would vary as a function of the emotion depicted in the context. Novel to this study, we predicted that context would exert stronger confusability effects for "open" disgust facial expressions than "closed" disgust facial expressions due to differences in perceptual similarity with the competing contextual cues and their corresponding facial expression.

\section{Method}

\section{Participants}

Thirty-one undergraduate students ( 14 female; $M_{\text {age }}=19.35$ years, $S D=1.38$ ) completed the study in exchange for course credit. Seventeen participants identified as Hispanic, 5 as Caucasian, 5 as African American, 3 as Asian, and 1 as mixed race. Previous studies using a similar design (Reschke et al., 2018) reported moderate to large effect sizes $\left(\eta_{p}^{2}=0.11-0.29\right)$ analogous to the confusability effects under examination here. Thus, our sample size was reasoned to have adequate power to detect similar confusability effects (i.e., .99-1.00, $\alpha=.05$, two tails).

\section{Stimuli}

Emotional stimuli consisted of facial expressions, emotion postures, and emotion scenes. All stimuli were validated independently to ensure that each component corresponded with the intended emotion.

Facial expressions. Four naturally posed facial expression images were taken from the NimStim set of facial expressions (Tottenham et al., 2009). The images included one male actor and one female actor each posing one "open" and one "closed" disgust facial expression. "Open" versions of disgust featured a wrinkled nose, dropped jaw, and protruding tongue. "Closed" versions of disgust featured a wrinkled nose and raised upper lips. Open and closed disgust expressions were previously validated as depicting disgust (female-open: 91\%; female-closed: 91\%; male-open: $96 \%$; male-closed: 91\%; Tottenham et al., 2009).

To further ensure the validity of the facial expressions, each image was separately evaluated by a coder trained in the Facial Action Coding System (FACS). The male and female closed disgust expressions were both coded as AUs $9+17$. The open disgust expressions were coded similarly across gender, though some small differences were observed. Specifically, the male actor expressed AUs $4+7+10+19 b+27$, whereas the female actor expressed AUs $1+4+10+19 b+26$. Importantly, the evaluation by the FACS coder characterized all facial expressions as disgust.

Emotion postures. Twelve emotion posture images were taken from previously validated sets of stimuli. The images included a male and female actor expressing five discrete emotions (disgust, anger, sadness, fear, joy; Lopez, Reschke, Knothe, \& Walle, 2017) and a neutral posture (Reschke et al., 2018). All postural expressions were highly validated (range: 76-100\%).

Emotion scenes. Thirty emotion scene images were validated for use in this study. Scene images consisted of 30 pictures depicting six emotions (disgust, anger, sadness, fear, joy, neutral) with five naturalistic exemplars (e.g., disgust = overflowing toilet; sadness $=$ funeral with casket) per emotion to minimize familiarization effects. All scene stimuli were accurately recognized as depicting the intended emotion (range: 76-100\%). Scene stimuli within emotion categories were also rated similarly in affective valence and arousal (see Russell, Weiss, \& Mendelsohn, 1989), thus allowing scenes to be collapsed by emotion category in the analyses (see online supplemental Table S1 for descriptions and validation information for the 30 scene exemplars).

Face-posture-scene composite images. Facial expressions (i.e., open and closed disgust faces) were superimposed onto congruent posture-scenes (e.g., a fear posture in a fear scene) using Adobe Photoshop to create 120 distinct face-posture-scene combinations ( 2 facial expressions $\times 2$ genders $\times 6$ posture-scenes $\times$ 5 scene exemplars; see Figure 1).

\section{Procedure}

The study took place in a campus computer lab with participants seated at individual computers with a 20 -inch monitor. Participants were randomly assigned to either the "open" condition $(n=16)$ or the "closed" condition $(n=15)$. All participants completed a demographics questionnaire. Participants then viewed the faceposture-scene composite images corresponding to their condition in a random order. Each set of 60 open or closed images was embedded in a larger set of 300 distinct and randomly presented face-posture-scene emotion filler images that were not relevant to the research aims of the study.

For each image, the participant was instructed to "select the emotion that best describes the facial expression." Below this prompt were the following options provided vertically in a set order: joy, sadness, fear, anger, disgust. Participants were given as much time as needed to respond. There was a 5-min break after participants rated half of the images. The entire survey took approximately $50 \mathrm{~min}$ to complete. Procedures were approved by the Institutional Review Board of the University of California, Merced.

\section{Results}

Participants' emotion categorizations organized by face type are provided in online supplemental Table S2. Participants' disgust categorizations (i.e., accuracy) and categorizations matching congruent postures and scenes (i.e., contextual influence) were analyzed separately using repeated-measures mixed-effects models specified with a binomial distribution, a log link function, and a compound symmetry covariance matrix. All models used restricted maximum likelihood. Each model included posture-scene (disgust, anger, fear, sadness, joy, neutral) as a within-subjects factor and facial configuration (open, closed) as a between-subjects factor, as well as the interaction term. ${ }^{2}$ Significant main effects were explored using Bonferroni-corrected pairwise comparisons. Prelim-

\footnotetext{
${ }^{2}$ An additional model analyzing all 360 images shown to each participant was examined that included face type, posture, scene, and all interactions. No differences in the effects and comparisons of interest were present. Thus, only the results for the specific open and closed disgust face images with the congruent posture-scenes are reported.
} 

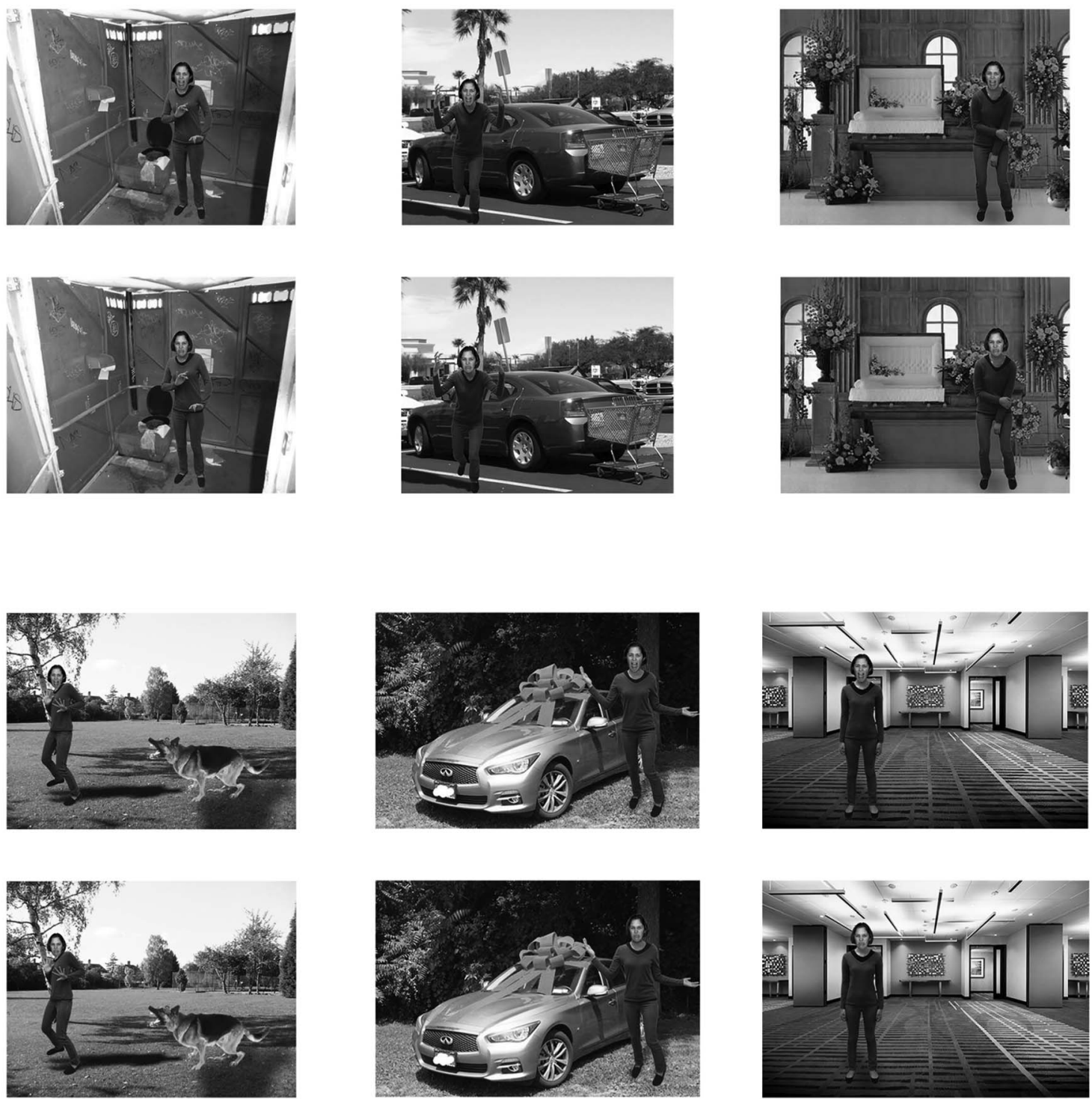

Figure 1. Examples of face-posture-scene stimuli. Order of contexts from top, left to right: disgust, anger, sadness, fear, joy, and neutral. Each vertical pair features an "open" disgust facial expression (top image) and "closed" disgust facial expression (bottom image). Facial expressions from the NimStim Set of Facial Expressions found at www.macbrain.org. Copyright by Nim Tottenham. Reproduced with permission.

inary analyses revealed no significant effects of participant gender, $p=.78$. Additionally, we examined the effect of actor to account for the subtle differences between the facial expressions displayed by each actor. No effect of actor was observed, $p=.83$. Thus, the participant gender and actor factors were collapsed in subsequent analyses.

\section{Accuracy}

We first analyzed participants' accuracy for categorizing disgust facial expressions. Results indicated significant effects of posturescene, $F(5,1,848)=64.35, p<.001, \eta_{p}^{2}=.15$, and facial configuration, $F(1,1,848)=5.41, p=.02, \eta_{p}^{2}=.003$, as well as a significant Posture-Scene $\times$ Facial Configuration interaction, $F(5,1,848)=2.57, p=.03, \eta_{p}^{2}=.01$ (see Table 1$)$.

Pairwise comparisons investigating the main effect of posturescene $(\alpha=.003)$ revealed that accuracy was significantly higher in disgust contexts than anger $(p=.001)$, sadness $(p=.001)$, fear $(p=.001)$, joy $(p=.001)$, and neutral $(p=.001)$ contexts. Additionally, accuracy was significantly higher in joy contexts than anger $(p=.001)$, sadness $(p=.001)$, and neutral $(p=.001)$ contexts and significantly higher in fear contexts than anger contexts $(p=.001)$. No other comparisons were statistically significant $(p s>.03)$.

Examination of the significant main effect of facial configuration $(\alpha=.05)$ revealed that participants were significantly less 
Table 1

Comparisons of Mean Accuracy and Contextual Influence Between Closed and Open Disgust Facial Expressions

\begin{tabular}{lccccccc}
\hline & & & & & & \multicolumn{2}{c}{$95 \%$ CI } \\
\cline { 6 - 8 } \multicolumn{1}{l}{ Posture-Scene } & Closed & Open & $t$ & $p$ & Cohen's $d$ & LL & UL \\
\hline Accuracy & & & & & & & \\
$\quad$ Disgust & .83 & .75 & 0.88 & .38 & 0.33 & -.09 & .25 \\
Anger & .22 & .04 & 2.50 & .01 & 0.93 & .04 & .32 \\
Sadness & .25 & .09 & 2.01 & .04 & 0.75 & .01 & .31 \\
Fear & .39 & .09 & 3.39 & .001 & 1.26 & .12 & .46 \\
Joy & .41 & .15 & 2.85 & .004 & 1.06 & .08 & .45 \\
$\quad$ Neutral & .23 & .08 & 1.91 & .06 & 0.71 & -.004 & .30 \\
Contextual influence & & & & & & & .25 \\
Disgust & .83 & .75 & 0.88 & .38 & 0.33 & -.09 & .004 \\
Anger & .69 & .86 & 1.92 & .06 & 0.71 & -.34 & .08 \\
Sadness & .73 & .83 & 1.11 & .27 & 0.41 & -.27 & .51 \\
Fear & .53 & .86 & 3.48 & .001 & 1.29 & -.51 & .05 \\
Joy & .11 & .32 & 2.52 & .01 & 0.94 & -.38 & -.05 \\
\hline
\end{tabular}

Note. $\quad \mathrm{LL}=$ lower limit; $\mathrm{UL}=$ upper limit.

accurate categorizing open disgust facial expressions than closed disgust facial expressions $(p=.01)$.

To test the primary question of interest, pairwise comparisons investigated the significant Posture-Scene $\times$ Facial Configuration interaction (see Table $1 ; \alpha=.05$ ). Participants were significantly less accurate in categorizing open disgust faces than closed disgust faces when embedded in contexts of anger $(p=.01)$, sadness $(p=$ $.04)$, fear $(p=.001)$, and joy $(p=.004)$. However, accuracy between open and closed disgust faces did not differ in the disgust $(p=.38)$ and neutral contexts $(p=.06)$. It should be noted that although these differences were not significant, they were in the same direction as the other significant comparisons.

\section{Contextual Influence}

Next, we examined participant emotion categorizations matching the posture-scene. Results indicated a significant main effect of posture-scene, $F(4,1,540)=68.28, p<.001, \eta_{p}^{2}=.15$, as well as a significant Posture-Scene $\times$ Facial Configuration interaction, $F(4,1,540)=11.11, p<.001, \eta_{p}^{2}=.03$ (see Table 1). However, the main effect of facial configuration was not significant, $F(1$, $1,540)=1.84, p=.09, \eta_{p}^{2}=.002$.

Further analysis of the main effect of posture-scene $(\alpha=.003)$ revealed that contextual influence was significantly lower in joy contexts than disgust ( $p=.001)$, anger $(p=.001)$, sadness $(p=$ $.001)$, and fear $(p=.001)$ contexts. No other comparisons reached statistical significance ( $p s \geq .05$ ).

As a test of our main hypothesis, pairwise comparisons investigating the significant Posture-Scene $\times$ Facial Configuration interaction $(\alpha=.05)$ revealed that categorizations matching the context were significantly higher for open disgust faces than closed disgust faces when embedded in joy $(p=.01)$ and fear contexts ( $p=.001$; see Table 1$)$. However, contextual influence between open and closed disgust faces did not differ significantly for disgust $(p=.38)$, anger $(p=.06)$, and sadness $(p=.27)$ contexts. Thus, joy and fear contexts elicited stronger confusability effects when combined with open disgust faces than closed disgust faces.

\section{Discussion}

The above findings replicate and extend prior research. Participant categorization of disgust facial expressions varied as a function of the accompanying context (i.e., body postures in emotion scenes). However, and novel to this study, disgust categorizations varied as a function of facial configuration, with open disgust facial expressions resulting in greater confusability effects than those observed for closed versions. Moreover, we observed significant increases in categorizations matching the fear and joy contexts, with fear resulting in a categorical change in emotion perception.

These findings advance our understanding of face perception in several ways. This is the first study to demonstrate that differences in physical configuration within discrete categories of affective facial expressions can result in varying confusability effects of face perception. As such, it is important that theories addressing contextual influences on emotion perception (e.g., Aviezer, Ensenberg, \& Hassin, 2017; Barrett, Mesquita, \& Gendron, 2011; Hassin et al., 2013) consider the effects of structural variation between and within discrete emotional facial expressions. For example, fear facial expressions are typically considered low in perceptual similarity with disgust facial expressions, whereas anger faces are high (Susskind et al., 2007). Accordingly, disgust facial expressions have been reported to be resistant to contextual influence when placed in fear contexts, yet responsive to contextual influence when placed in anger contexts (e.g., Aviezer et al., 2008). However, the current results demonstrated that "open" disgust facial expressions were equally influenced by fear contexts and anger contexts, with both contexts producing confusability effects. The perceptual similarity hypothesis contends that physical similarities between discrete emotion facial expressions leads to varied confusability effects when facial expressions are perceived in context. However, the present findings encourage a more nuanced perspective. Specifically, the physical similarity between discrete facial expressions appears to be more flexible than fixed, resulting in confusability effects that vary as a function of particular withincategory configurations of facial action units. Moreover, such 
confusability effects are likely dependent on within-category differences in emotion contexts.

\section{Limitations and Future Directions}

Although informative for the field of emotion perception, the current findings were limited to disgust facial expressions. Other emotion facial expressions also have varying levels of structural flexibility. For example, fear and surprise facial expressions share more physical similarities than differences (Ekman, 2003), and slight changes in one expression can result in a categorical shift between these expressions (Roy-Charland, Perron, Beaudry, \& Eady, 2014). Research is needed to examine how structural variability within additional discrete categories of facial expressions is affected by and interacts with contextual elements.

Additionally, our use of open- and closed-mouthed versions of disgust facial expressions represents only two possible structural configurations of disgust. However, disgust can be conveyed in other ways not captured by the present stimuli. Research suggests that the mouth region plays a diagnostic role in differentiating disgust from other facial expressions (Wegrzyn, Vogt, Kireclioglu, Schneider, \& Kissler, 2017). Further research is needed to examine how the addition of nondiagnostic emotion cues for one emotion (e.g., a furrowed brow for disgust) may lead to increased confusability with other expressions for which the cue is diagnostic (e.g., a furrowed brow for anger; Pochedly, Widen, \& Russell, 2012; Smith, Cottrell, Gosselin, \& Schyns, 2005). Moreover, research examining how individuals visually process such facial cues in context would provide a clearer picture of how individuals perceive and make sense of combinations of emotional elements. Additionally, it is possible that observed differences in contextual effects were due to differences in the actors used for the stimuli instead of face type, although this alternative explanation is unlikely given the null effect of actor. Even so, as the present study only used two face actors, further research including multiple actors within emotion facial expression would strengthen the current findings.

Finally, the "open" and "closed" versions of disgust facial expressions in the present study were naturally posed (Tottenham et al., 2009) and not created using predetermined configurations of AUs (e.g., Ekman \& Friesen, 1978). Research using more structurally standardized facial expressions is needed to confirm our results, as well as further explore how specific AUs may interact with contextual elements. However, such studies must work to ensure that emotional stimuli are ecologically valid, a point frequently advocated in emotion perception research (e.g., Matsumoto \& Hwang, 2010; Russell, 1994).

\section{Conclusion}

Emotion perception is a multifaceted process that involves perceiving the gestalt of multiple emotionally relevant cues. These results suggest that face perception is susceptible to varying levels of contextual influence as a function of the structural variability within a given facial expression. Indeed, face and context appear to be interactive cues for emotion perception. We urge continued research on perceiving emotions in context and how various emotional cues may be additive and interactive in their effects.

\section{References}

Aviezer, H., Ensenberg, N., \& Hassin, R. R. (2017). The inherently contextualized nature of facial emotion perception. Current Opinion in Psychology, 17, 47-54. http://dx.doi.org/10.1016/j.copsyc.2017.06.006

Aviezer, H., Hassin, R. R., \& Bentin, S. (2012). Impaired integration of emotional faces and affective body context in a rare case of developmental visual agnosia. Cortex: A Journal Devoted to the Study of the Nervous System and Behavior, 48, 689-700. http://dx.doi.org/10.1016/ j.cortex.2011.03.005

Aviezer, H., Hassin, R. R., Ryan, J., Grady, C., Susskind, J., Anderson, A., . . . Bentin, S. (2008). Angry, disgusted, or afraid? Studies on the malleability of emotion perception. Psychological Science, 19, 724-732. http://dx.doi.org/10.1111/j.1467-9280.2008.02148.x

Aviezer, H., Trope, Y., \& Todorov, A. (2012). Body cues, not facial expressions, discriminate between intense positive and negative emotions. Science, 338, 1225-1229. http://dx.doi.org/10.1126/science.1224313

Barrett, L. F., Mesquita, B., \& Gendron, M. (2011). Context in emotion perception. Current Directions in Psychological Science, 20, 286-290. http://dx.doi.org/10.1177/0963721411422522

Bliss-Moreau, E., Moadab, G., \& Machado, C. J. (2017). Monkeys preferentially process body information while viewing affective displays. Emotion, 17, 765-771. http://dx.doi.org/10.1037/emo0000292

Calder, A. J., Keane, J., Young, A. W., Lawrence, A. D., Mason, S., \& Barker, R. A. (2010). The relation between anger and different forms of disgust: Implications for emotion recognition impairments in Huntington's disease. Neuropsychologia, 48, 2719-2729. http://dx.doi.org/10 .1016/j.neuropsychologia.2010.05.019

Ekman, P. (1993). Facial expression and emotion. American Psychologist, 48, 384-392. http://dx.doi.org/10.1037/0003-066X.48.4.384

Ekman, P. (2003). Emotions revealed: Recognizing faces and feelings to improve communication and emotional life. New York, NY: Times Books.

Ekman, P., \& Friesen, W. (1978). The facial action coding system: A technique for the measurement of facial movement. Palo Alto, CA: Consulting Psychologists Press.

Ekman, P., \& O'Sullivan, M. (1988). The role of context in interpreting facial expression: Comment on Russell and Fehr (1987). Journal of Experimental Psychology: General, 117, 86-88. http://dx.doi.org/10 .1037/0096-3445.117.1.86

Hassin, R. R., Aviezer, H., \& Bentin, S. (2013). Inherently ambiguous: Facial expressions of emotions, in context. Emotion Review, 5, 60-65. http://dx.doi.org/10.1177/1754073912451331

Lopez, L. D., Reschke, P. J., Knothe, J. M., \& Walle, E. A. (2017). Postural communication of emotion: Perception of distinct poses of five discrete emotions. Frontiers in Psychology, 8, 710. http://dx.doi.org/10.3389/ fpsyg.2017.00710

Matsumoto, D., \& Sung Hwang, H. (2010). Judging faces in context. Social and Personality Psychology Compass, 4, 393-402.

Meeren, H. K., van Heijnsbergen, C. C., \& de Gelder, B. (2005). Rapid perceptual integration of facial expression and emotional body language. Proceedings of the National Academy of Sciences of the United States of America, 102, 16518-16523. http://dx.doi.org/10.1073/pnas.05076 50102

Mondloch, C. J., Nelson, N. L., \& Horner, M. (2013). Asymmetries of influence: Differential effects of body postures on perceptions of emotional facial expressions. PLOS ONE, 8, e73605. http://dx.doi.org/10 .1371 /journal.pone. 0073605

Pochedly, J. T., Widen, S. C., \& Russell, J. A. (2012). What emotion does the "facial expression of disgust" express? Emotion, 12, 1315-1319. http://dx.doi.org/10.1037/a0027998

Reschke, P. J., Knothe, J. M., Lopez, L. D., \& Walle, E. A. (2018). Putting "context" in context: The effects of body posture and emotion scene on adult categorizations of disgust facial expressions. Emotion, 18, 153158. http://dx.doi.org/10.1037/emo0000350 
Roy-Charland, A., Perron, M., Beaudry, O., \& Eady, K. (2014). Confusion of fear and surprise: A test of the perceptual-attentional limitation hypothesis with eye movement monitoring. Cognition and Emotion, 28, 1214-1222. http://dx.doi.org/10.1080/02699931.2013.878687

Rozin, P., Lowery, L., \& Ebert, R. (1994). Varieties of disgust faces and the structure of disgust. Journal of Personality and Social Psychology, 66, 870-881. http://dx.doi.org/10.1037/0022-3514.66.5.870

Russell, J. A. (1994). Is there universal recognition of emotion from facial expression? A review of the cross-cultural studies. Psychological Bulletin, 115, 102-141. http://dx.doi.org/10.1037/0033-2909.115.1.102

Russell, J. A., Weiss, A., \& Mendelsohn, G. A. (1989). Affect grid: A single-item scale of pleasure and arousal. Journal of Personality and Social Psychology, 57, 493-502. http://dx.doi.org/10.1037/0022-3514 .57.3.493

Smith, M. L., Cottrell, G. W., Gosselin, F., \& Schyns, P. G. (2005). Transmitting and decoding facial expressions. Psychological Science, 16, 184-189. http://dx.doi.org/10.1111/j.0956-7976.2005.00801.x

Susskind, J. M., Littlewort, G., Bartlett, M. S., Movellan, J., \& Anderson, A. K. (2007). Human and computer recognition of facial expressions of emotion. Neuropsychologia, 45, 152-162. http://dx.doi.org/10.1016/j .neuropsychologia.2006.05.001
Tomkins, S. S., \& McCarter, R. (1964). What and where are the primary affects? Some evidence for a theory. Perceptual and Motor Skills, 18, 119-158. http://dx.doi.org/10.2466/pms.1964.18.1.119

Tottenham, N., Tanaka, J. W., Leon, A. C., McCarry, T., Nurse, M., Hare, T. A., . . Nelson, C. (2009). The NimStim set of facial expressions: Judgments from untrained research participants. Psychiatry Research, 168, 242-249. http://dx.doi.org/10.1016/j.psychres.2008.05.006

Wegrzyn, M., Vogt, M., Kireclioglu, B., Schneider, J., \& Kissler, J. (2017). Mapping the emotional face. How individual face parts contribute to successful emotion recognition. PLOS ONE, 12, e0177239. http://dx.doi .org/10.1371/journal.pone.0177239

Widen, S. C., Pochedly, J. T., Pieloch, K., \& Russell, J. A. (2013). Introducing the sick face. Motivation and Emotion, 37, 550-557. http:// dx.doi.org/10.1007/s11031-013-9353-6

Received September 14, 2017 Revision received February 28, 2018

Accepted March 1, 2018 